\title{
Genetic polymorphisms of phase II metabolic enzymes and lung cancer susceptibility in a population of Central South China ${ }^{1}$
}

\author{
Han-chun Chen, ${ }^{\mathrm{a}, *}$, Yan-fei Cao ${ }^{\mathrm{a}}$, Wei-xin $\mathrm{Hu}^{\mathrm{b}}$, Xin-fa Liu ${ }^{\mathrm{b}}$, Qing-xia Liu ${ }^{\mathrm{b}}$, Ji Zhang ${ }^{\mathrm{b}}$ and Jia Liu ${ }^{\mathrm{a}}$ \\ ${ }^{a}$ Department of Biochemistry, Xiangya School of Medicine, Central South University, Changsha 410078, Hunan, \\ China \\ ${ }^{\mathrm{b}}$ Molecular Biology Research Center, Xiangya School of Medicine, Central South University, Changsha 410078, \\ Hunan, China
}

\begin{abstract}
A case-control study was conducted for analyzing the genetic polymorphisms of phase II metabolic enzymes in 97 patients with lung cancer and 197 healthy subjects from Han ethnic group of Hunan Province located in Central South China. The results showed that the frequencies of glutathione S-transferase (GST) M1-null (GSTM1-) or GSTT1-null (GSTT1-) genotype alone, or combined form of both in lung cancer patients were significantly higher than those of the controls. Genotypes of combining GSTP1 mutant/GSTM1(-) or GSTP1 mutant/GSTT1(-) led to high risk of lung cancer. Individuals carrying any two or all three of GSTM1(-), GSTT1(-) and GSTP1 mutant genotypes have a distinctly increased risk of lung cancer when compared to those with GSTM1 present (GSTM1+: GSTM1+/+ or GSTM1+/-), GSTT1 present (GSTT1+: GSTT1+/+ or GSTT1+/-) and GSTP1 wild genotypes. Furthermore, individuals possessing combined genotypes of N-acetyltransferase 2 (NAT2) rapid acetylator, GSTP1 mutant and both GSTT1(-) and GSTM1(-) have a remarkably higher lung cancer risk than those carrying combined NAT2 slow acetylator genotype, GSTP1 wild genotype and both GSTT1(+) and GSTM1(+) genotypes. All these findings suggest that the genetic polymorphisms of phase II metabolic enzymes affect the susceptibility of lung cancer in the Han ethnic group of Central South China.
\end{abstract}

Keywords: Genetic polymorphism, GST, NAT2, lung cancer, susceptibility

\section{Introduction}

Both environmental and genetic factors are considered important in the etiology of human tumors. The risk of cancer correlated with exposures to exogenous xenobiotics or endogenous substances may be modified by genetic variation in metabolic detoxification activ-

\footnotetext{
${ }^{1}$ The work was supported by the grant No.30371660 from the National Natural Science Foundation of China and the grant CMB No. 99-698 from China Medical Board of New York, INC.

${ }^{*}$ Corresponding author: Han-chun Chen, MD., Ph.D., Department of Biochemistry, Xiangya School of Medicine, Central South University, 110 Xiangya Road, Changsha, Hunan, 410078, P.R. China. Tel.: +86 731 2650415; +86 731 4918173; Fax: +86 731 2650230; E-mail: chenhanchun@mail.csu.edu.cn.
}

ities [1]. Phase II biotransformation enzymes generally act as inactivating enzymes to catalyze the binding of intermediary metabolites to cofactors, transform them into more hydrophilic products and thus facilitate their elimination. Both GSTs and NATs are phase II transformation enzymes involved in the detoxification of hazardous agents [2]. GSTM1, GSTT1 and GSTP1 are dimeric enzyme members constituting GST super-family that catalyze the conjugation of glutathione to biotransform toxic chemicals into nontoxic substances [3]. The GSTM1 gene is polymorphic and is represented by two active alleles and a nonfunctional null allele which resulting from the entire GSTM1 gene deletion mutation. GSTM1 may act as a determinant factor in susceptibility to the related disease and may be a risk factor for cancer [4]. In humans, 
GSTT1 is polymorphic and represented by a functional (wild) allele and a non-functional (null) allele. This null allele results from total or partial deletion of the gene and presents two possible phenotypes: GSTT1 null, which is the homozygote of the deleted allele, and GSTT1-positive, which is the phenotype that at least one copy of the gene is intact [5]. GSTT1 null phenotype appears to increase the susceptibility to some types of cancer [6]. GSTP1 alleles have been described in four types, the wild-type allele and three variant alleles. These different alleles result from the combination of two single nucleotide polymorphisms (SNPs) within the GSTP1 gene, one of which resulting in a change at codon 105 (Ile/Val), and the other at codon 114 (Ala/Val). The wild-type allele has Ile at codon 105 and Ala at codon 114. However, the residue at codon 105 lies in close proximity to the hydrophobic binding site of electrophilic substrates [7] and the $105 \mathrm{Val}$ variant has altered specific activity and affinity for electrophilic substrates [8]. Watson et al. [9] investigated the genotype at exon 5 and exon 6 of the human GSTP1 gene in normal lung tissue obtained from surgical patients and found that the Ala114Val polymorphism in exon 6 was less common than the Ile105Val in population, and there was a non-significant trend toward lower mean GST enzyme activity among individuals with the 114 valine allele but GST activities in lung tissue were significantly lower among individuals with the 105Val allele. Thus, the polymorphism at codon 105 of human GSTP1 gene results in active proteins with different enzyme activity. The polymorphisms of human GSTP1 gene for codon 105 present three genotypes: wild genotype (AA, Ile/Ile), heterozygous mutant genotype (AG, Ile/Val) and homozygous mutant genotype (GG, Val/Val). GSTP1 enzymes with 105Val allele showed different catalytic efficiency for various chemicals or drugs [10]. Individuals with the 105Val allele have a higher risk of developing lung cancer than individuals with the 105Ile allele [11].

NATs catalyze the activation (O-acetylation) of heterocyclic amine carcinogens and are subject to genetic polymorphism [12]. In humans, there are two active genes coding NATs, NAT1 and NAT2, and one noncoding NATP pseudogene [13]. Excepting the wildtype allele (NAT2*4), the other 25 variants of NAT2 alleles possess a combination of one to four SNPs at 11 sites within the $870 \mathrm{bp}$ coding region. The NAT2 polymorphism is very common in the human population and the phenotypes of individuals can be subdivided into three groups, homozygous rapid (with a high O-acetylation capacity), heterozygous rapid (or inter- mediate) and slow acetylator [14]. The correlation of NAT2 genotype with lung cancer risk is quite different in different populations [15-18].

Lung cancer is a major scourge in our time and remains a worldwide healthcare problem. In the past two decades, the mortality of lung tumor has been doubled in Hunan population [19]. In human lung, GSTT1, GSTP1, and, to a lesser extent, GSTM1 are present in tissue [20-22], and NAT2 is expressed mainly in the epithelial lining of the bronchi [23]. Thus it is of great importance to evaluate genetic polymorphism of both GST members and NAT2 together as putative factors affecting the susceptibility of human lung cancer. Individuals carrying more than one of the risking polymorphisms may have a greater risk of developing lung cancer [24].

It has been proved that cigarette smoking is an obvious effective modifier, which increases the risk of lung cancer development. However, only $10-15 \%$ of smokers did develop lung cancer when exposed to a variety of xenobiotics from both inhalational and noninhalational routes. This may be explained as that hereditary, environmental, occupational, and dietary factors are involved in influencing the development of lung cancer. In addition to tobacco smoking, genetic damage caused by a broad range of chemical carcinogens from the contamination of food, water, living and working air environment, is also considered as the factor that causes the increase of lung cancer risk [25]. For example, lung adenocarcinoma is the most common cell type in females (smokers or non-smokers) and in non-smoking males [26]. The rate of lung cancer in China marks one of the highest in the world, mainly including squamous cell carcinoma and adenocarcinoma in non-smoking women $[27,28]$. It has been reported that long-term occupational exposure to environmental tobacco smoke (ETS), both alone or in combination with exposures at home, conferred an increased risk of lung cancer among Chinese women who never smoked in Shanghai area [29]. Yang et al. [30] reported that CYP1A1 Ile462Val genotype (combined Ile/Val and Val/Val) was significantly associated with lung cancer risk in a Chinese population, but the GSTM1 null genotype did not. After analyzing combined genotypes, $\mathrm{Ng}$ et al. [31] concluded that CYP1A1 is a susceptibility gene for lung cancer among non-smoking Asian women and this association can be influenced by ETS exposure and genetic variation of GST genes. The finding of Nakajima et al. [32] that there is no significant difference in GSTP1 activity in lung tissue among samples from smokers and non-smokers provided the direct 
evidence that tobacco may not be an effective inducer of GSTP1 in lung.

The enzyme NAT2 catalyzes the acetylation of arylamines which are ubiquitous chemicals present in industry, cooked food and as environmental contaminants [33]. It has been reported that slow NAT2 acetylator genotype associated with increased risk of lung cancer in female non-smokers [18]. However, Chiou et al. [34] found that NAT2 fast acetylator genotype was associated with an increased risk of lung cancer among never-smoking women in Taiwan. These conflicting results also suggested that cigarette smoking cannot fully explain the epidemiologic characteristics of lung cancer, and some other factors, including hereditary and other environmental factors, may be also involved in lung cancer development [35]. To evaluate the risk factors of lung cancer in non-smoking Chinese population, we epidemiologically investigated the relationship of genetic polymorphisms of phase II metabolic enzyme family members GSTM1, GSTT1, GSTP1 and NAT2 and risk of lung cancer in non-smoking Han ethnic population in Hunan Province of China by PCR and PCR-RFLP analysis for the first time. The combined genotypes analysis for phase II metabolic enzymes family members might provide a rational strategy for lung cancer risk estimation and prevention in Han ethnic population of Central South China.

\section{Materials and methods}

\subsection{Grouping of subjects}

This case-control study included 97 cases with lung cancer and 197 cancer-free and lung disease-free healthy controls. All subjects were selected from Han ethnic group born and living in Hunan Province. The patients were all pathologically confirmed as primary lung cancer at Xiangya Hospital, the affiliated teaching hospital of Central South University in Changsha City, Hunan Province. 55 among them were females and 42 were males, with an average age of 56.6 years old. They were non-smokers and mostly suffered from squamous cell carcinoma ( $n=51,52.6 \%$ ) or adenocarcinoma ( $n=43,44.3 \%$ ), except 3 cases that the subtypes were undetermined. The 197 controls were recruited from citizens taking part in a health-screening survey carried out at Xiangya School of Medicine. All of them were also enrolled from non-smokers and matched by sex and age (101 females and 96 males, with an average age of 55.8 years old) to the patients.

\subsection{Determination of genetic polymorphisms}

$10 \mathrm{ml}$ of heparinized peripheral blood were collected from each individual and stored at $-80^{\circ} \mathrm{C}$. Genomic DNA was purified from the thawed blood samples by using standard SDS/proteinase K treatment and phenol-chloroform extraction. The purified DNA samples were dissolved in Tris-EDTA buffer (TE buffer, $\mathrm{pH}$ 7.4) and adjusted the concentration to $100 \mathrm{ng} / \mu \mathrm{l}$ by estimation of absorbance at $260 \mathrm{~nm}$. Quality of the DNA sample was assessed by measurement of the A260 nm/A280 nm absorbance ratio $(1.75 \sim 1.95)$ and agarose gel electrophoresis. DNA samples were stored in TE buffer at $-20^{\circ} \mathrm{C}$ till PCR analysis. $1 \mu \mathrm{l}$ (100 ng) of each crude DNA preparation was used for per PCR reaction.

The allele sequences of GSTs and NAT2 polymorphic variants were searched from Genbank. PCR primers (appendix 1) for the various alleles of GSTM1, GSTT1, GSTP1 and NAT2 variants were designed by using software Primer Preimer 5 according to the template sequence. PCR was carried out in a total volume of $50 \mu \mathrm{l}$ containing $100 \mathrm{ng}$ of DNA template, 10 pmole/L of each forward $(\mathrm{F})$ and reverse $(\mathrm{R})$ primers, $200 \mu$ mole/L of each dNTP and 2 units of Taq polymerase (Roche). The mixture was initially incubated for $5 \mathrm{~min}$ at $94^{\circ} \mathrm{C}$ to denature the template DNA; And then the PCR reactions were carried out for 30 cycles under the conditions of denaturation for $30 \mathrm{~s}$ at $94{ }^{\circ} \mathrm{C}$, annealing for $30 \mathrm{~s}$ at the optimal temperature (Appendix 1), and extension for $45 \mathrm{~s}$ at $72^{\circ} \mathrm{C}$, and a final extension period of $5 \mathrm{~min}$ at $72^{\circ} \mathrm{C}$ was performed after the PCR cycles finished. $6 \mu \mathrm{l}$ aliquot of PCR products was loaded on a $1.5 \%$ agarose gel containing $0.5 \mathrm{mg} / \mathrm{ml}$ of ethidium bromide to separate the DNA fragments. The DNA bands were visualized and photographed under UV light after electrophoresis.

The absence of GSTM1 or GSTT1 specific fragment indicated the corresponding null genotype, meanwhile using $\beta$-actin specific primers to amplify the specific fragment as internal control to confirm the presence of amplifiable DNA template in the reaction mixture. In order to further verify the gene deletion, a second PCR was performed by using another pair of PCR primers in extension for the same gene.

In order to detect the mutation in GSTP1 and NAT2 allele variants, the related PCR product was digested separately with Alw26 I (for GSTP1 mutation site), Taq I, Msp I, Fok I, Aci I, Kpn I, Dde I, Dra III and BamH I (NAT2 mutation sites showed in Appendix 2) to 
Table 1

Demographic characteristics of the lung cancer cases and controls

\begin{tabular}{lcc}
\hline Characteristics & Cases $(n=97)$ & Controls $(n=197)$ \\
\hline Gender, $n(\%)$ & & \\
Male & $42(43.3)$ & $96(48.7)$ \\
Female & $55(56.7)$ & $101(51.3)$ \\
Age range & $28-72$ & $31-69$ \\
Mean age & 56.6 & 55.8 \\
Subtype of cancer, $n(\%)$ & & \\
Squamous cell & $51(52.6)$ & \\
Adenocarcinoma & $43(44.3)$ & \\
Non-classified & $3(3.1)$ & \\
\hline
\end{tabular}

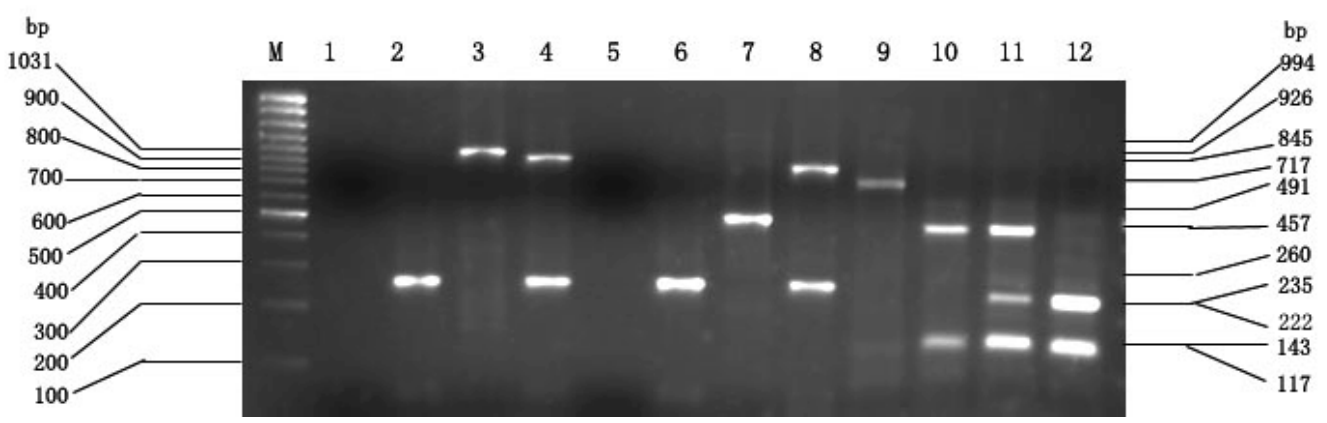

Fig. 1. Agarose gel electrophoresis of PCR products from GST genes and their restriction fragments. M. 100 bp DNA ladder Marker; 1. GSTM1-F1/R1 (deletion); 2. GSTM1-F2/R2 (deletion)/ $\beta$-actin; 3. GSTM1-F2/R2; 4. GSTM1-F1/R1/ $\beta$-actin; 5. GSTT1-F1/R1(deletion); 6. GSTT1-F2/R2 (deletion)/ $\beta$-actin; 7. GSTT1-F2/R2; 8. GSTT1-F1/R1/ $\beta$-actin; 9. GSTP1-F1/R1; 10 . GSTP1-F1/R1(Alw26 I)/Wild; 11 . GSTP1-F1/R1(Alw26 I)/Heterozygous mutant; 12. GSTP1-F1/R1(Alw26 I)/Homozygous mutant.

analyze the restriction fragment length polymorphism (RFLP), respectively.

$10 \mu \mathrm{l}$ of PCR product and 10 units of restriction enzyme were used for each digestion reaction. All the reactions were carried out overnight at $37^{\circ} \mathrm{C}$, except Taq I and Fok I which were performed at $65^{\circ} \mathrm{C}$ and $55^{\circ} \mathrm{C}$ respectively. All digested products were separated and analyzed on ethidium bromide-stained $2 \%$ or $3 \%$ agarose gels. For PCR/RFLP analysis, the corresponding sequenced PCR products were used as positive or negative controls.

\section{Statistical methods}

Allele and genotype frequencies were reported as percentages. The difference in frequencies between patients and healthy controls was compared using $\chi^{2}$ test. A value of $P<0.05$ was considered statistically significant. Odds ratios (OR) and $95 \%$ confidence intervals $(95 \% \mathrm{CI})$ were calculated by $\chi^{2}$ test, Fisher's Exact Test and unconditional multivariate logistic regression for comparing the difference of genotypes between patients and healthy controls, thus the correlation between genetic polymorphisms and lung cancer risk was identified. All calculations were accomplished with the SPSS version 11.5 statistical package.

\section{Results}

The demographic characteristics of the lung cancer cases and healthy controls were shown in Table 1 .

PCR products and the restriction map of GSTs and NAT2 genes were shown in Figs 1 to 3.

The distributions of three GSTs and NAT2 genotypes in lung cancer patients and healthy subjects were shown in Table 2. The frequencies of GSTM1(-) and GSTT1(-) genotypes significantly elevated in lung cancer patients $(61.9 \%$ and $60.8 \%$, respectively) as compared to those in health controls $(45.2 \%, P=0.007$ and $43.1 \%, P=0.004$, respectively). Both of the GSTM1() and GSTT1(-) genotypes were correlated with an increased risk of lung cancer $(\mathrm{OR}=1.968,95 \% \mathrm{CI}=$ $1.198-3.233$ and $\mathrm{OR}=2.046,95 \% \mathrm{CI}=1.246-3.358$, respectively). No obvious variation in frequencies of GSTP1 genotype (AA and AG+GG) and NAT2 genotype (slow and rapid acetylator) existed between patients and healthy controls $(P>0.05)$, but a distinct correlation was observed between lung cancer and the 


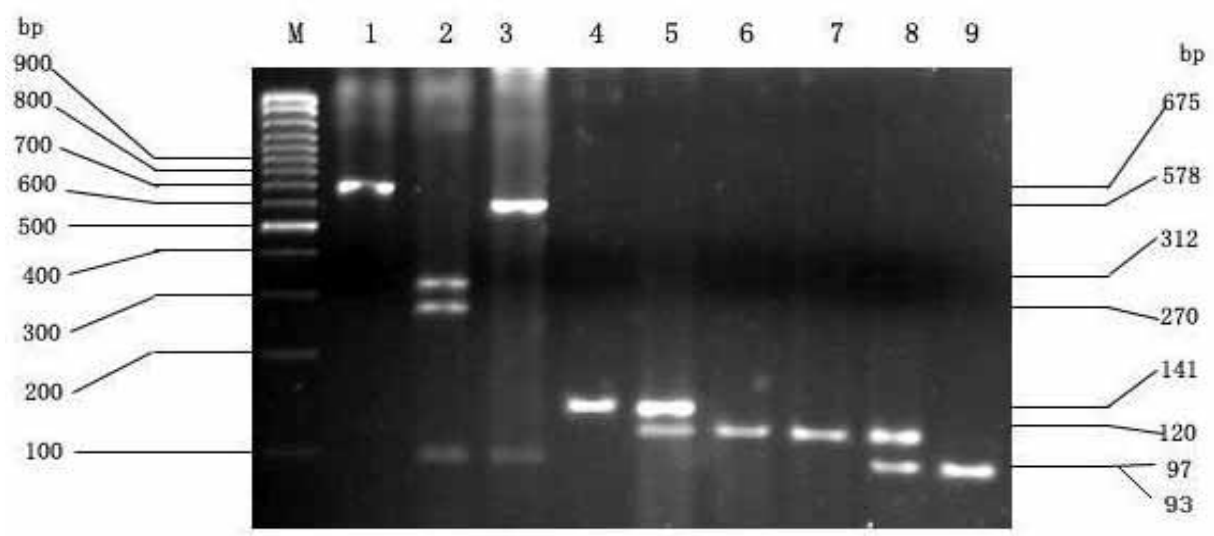

Fig. 2. Agarose gel electrophoresis of PCR products from NAT2 gene and their restriction fragments M. 100 bp DNA ladder Marker; 1. NAT2-F1/R1; 2. NAT2-F1/R1 (Msp I)/Wild; 3. NAT2-F1/R1 (Taq I)/Wild; 4. NAT2-F3/R1(Aci I)/Wild; 5. NAT2-F3/R1(Aci I)/Heterozygous mutant; 6. NAT2-F3/R1(Aci I)/Homozygous mutant; 7. NAT2-F4/R3(Dde I)/Wild; 8. NAT2-F4/R3(Dde I)/Heterozygous mutant; 9. NAT2-F4/R3(Dde I)/Homozygous mutant.

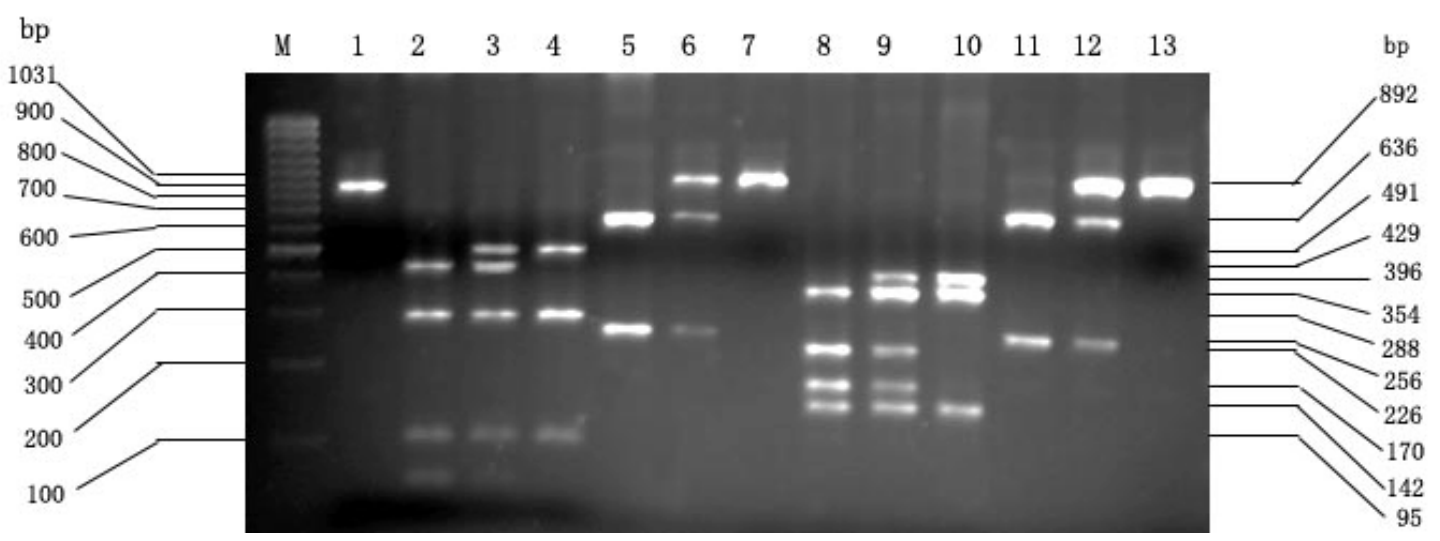

Fig. 3. Agarose gel electrophoresis of PCR products from NAT2 gene and their restriction fragments M. 100 bp DNA ladder Marker; 1. NAT2-F2/R2(Dra III)/ Wild; 2. NAT2-F2/R2(Fok I)/Wild; 3. NAT2-F2/R2(Fok I)/Heterozygous mutant; 4. NAT2-F2/R2(Fok I)/Homozygous mutant; 5. NAT2-F2/R2 (Kpn I)/Wild; 6. NAT2-F2/R2 (Kpn I)/Heterozygous mutant; 7. NAT2-F2/R2 (Kpn I)/Homozygous mutant; 8. NAT2-F2/R2 (Taq I)/Wild; 9. NAT2-F2/R2 (Taq I)/Heterozygous mutant; 10. NAT2-F2/R2 (Taq I)/Homozygous mutant; 11. NAT2-F2/R2 $($ BamH I)/Wild; 12. NAT2-F2/R2 (BamH I)/Heterozygous mutant; 13. NAT2-F2/R2 (BamH I)/Homozygous mutant.

genotypes of combination of GSTM1(-) and GSTT1(-) $(P=0.000)$, GSTP1(AG $+\mathrm{GG})$ and GSTM1(-) $(P=$ 0.006), as well as GSTP1(AG+GG) and GSTT1(-) $(P=0.003)$.

Combination studies of all three GSTs revealed that people carrying any two or all three of GSTM1(), GSTT1(-) and GSTP1(AG+GG) mutant genotypes possess prominently increased risk of lung cancer as compared to those with GSTM1 and GSTT1 present as well as GSTP1(AA) wild genotypes (Table 3).

NAT2 genotypes were classified into rapid acetylators (including homozygous and heterozygous fast acetylators) and slow acetylators. Individuals possessing NAT2 genotypes were correspondingly classified into three groups, homozygous rapid acetylators who possessing two NAT2 alleles associated with high acetylation activity (NAT2*4, NAT2* 12 or NAT2*13), heterozygous or intermediate acetylators who possessing one of these alleles, and slow acetylators who possessing none of these alleles [36]. As showed in Tables 2 and 4, distribution of NAT2 genotypes did not correlate with the risk of lung cancer significantly as compared to the healthy controls (OR $=1.225,95 \% \mathrm{CI}=0.664-2.263, P$ for trend $=$ $\left.0.516, \chi^{2}=0.423\right)$. However, individuals possessing combining genotypes of NAT2 rapid (homozygous fast/heterozygous fast or intermediate acetylators) and GSTM1(-), GSTT1(-) and GSTP1(AG+GG) mutant 
Table 2

Distribution of GSTs and NAT2 genotypes in lung cancer cases and controls

\begin{tabular}{|c|c|c|c|c|c|}
\hline Genotype & Cases $(\%)$ & Controls $(\%)$ & OR $(95 \% \mathrm{CI})$ & $P$ & $\chi^{2}$ \\
\hline \multicolumn{6}{|l|}{ GSTM1 } \\
\hline Present & $37(38.1)$ & $108(54.8)$ & & & \\
\hline Null & $60(61.9)$ & $89(45.2)$ & $1.968(1.198-3.233)$ & $0.007^{1}$ & 7.233 \\
\hline \multicolumn{6}{|l|}{ GSTT1 } \\
\hline Present & $38(39.2)$ & $112(56.9)$ & & & \\
\hline Null & $59(60.8)$ & $85(43.1)$ & $2.046(1.246-3.358)$ & $0.004^{1}$ & 8.128 \\
\hline \multicolumn{6}{|l|}{ GSTP1 } \\
\hline AA (Ile/Ile) & $66(68.0)$ & $143(72.6)$ & & & \\
\hline \multicolumn{6}{|l|}{$\mathrm{AG}+\mathrm{GG}$} \\
\hline (Ile/Val+Val/Val) & $31(32.0)$ & $54(27.4)$ & $1.244(0.733-2.112)$ & $0.419^{1}$ & 0.654 \\
\hline \multicolumn{6}{|l|}{ NAT2 } \\
\hline Slow & $18(18.6)$ & $43(21.8)$ & & & \\
\hline Rapid & $79(81.4)$ & $154(78.2)$ & $1.225(0.664-2.263)$ & $0.516^{1}$ & 0.423 \\
\hline GSTM1 (+)/GSTT1 (+) & $14(14.4)$ & $67(34.0)$ & & & \\
\hline GSTM1 (+)/GSTT1 (-) & $23(23.7)$ & $41(20.8)$ & $2.685(1.243-5.797)$ & $0.011^{2}$ & 6.545 \\
\hline GSTM1 (-)/GSTT1 (+) & $24(24.7)$ & $45(22.8)$ & $2.552(1.194-5.456)$ & $0.014^{2}$ & 6.032 \\
\hline GSTM1 (-)/GSTT1 (-) & $36(37.1)$ & $44(22.3)$ & $3.916(1.896-8.085)$ & $0.000^{2}$ & 14.44 \\
\hline GSTP1 (AA)/GSTM1 (+) & $26(26.8)$ & 74 (37.6) & & & \\
\hline GSTP1 (AA)/GSTM1 (-) & $40(41.2)$ & $69(35.0)$ & $1.650(0.912-2.985)$ & $0.097^{3}$ & 2.762 \\
\hline \multicolumn{6}{|l|}{ GSTP1 (AG+GG)/ } \\
\hline GSTM1 (+) & $11(11.3)$ & $34(17.3)$ & $0.921(0.480-2.077)$ & $0.842^{3}$ & 0.040 \\
\hline GSTM1 (-) & $20(20.6)$ & $20(10.2)$ & $2.846(1.326-6.111)$ & $0.006^{3}$ & 7.460 \\
\hline GSTP1 (AA)/GSTT1 (+) & $31(32.0)$ & $79(40.1)$ & & & \\
\hline GSTP1 (AA)/GSTT1 (-) & $35(36.1)$ & $64(32.5)$ & $1.394(0.776-2.502)$ & $0.265^{4}$ & 1.240 \\
\hline \multicolumn{6}{|l|}{ GSTP1 (AG+GG)/ } \\
\hline GSTT1 (+) & $7(7.2)$ & $33(16.8)$ & $0.541(0.216-1.350)$ & $0.183^{4}$ & 1.769 \\
\hline GSTT1 (-) & $24(24.7)$ & $21(10.7)$ & $2.912(1.420-5.971)$ & $0.003^{4}$ & 8.825 \\
\hline
\end{tabular}

${ }^{1}$ Comparison between lung cancer patients and healthy controls.

${ }^{2}$ Comparison between GSTM1 $(+) /$ GSTT1 $(+)$ and the other combined genotypes of GSTM1/GSTT1 in relation to lung cancer.

${ }^{3}$ Comparison between GSTP1 (AA)/GSTM1 (+) and the other combined genotypes of GSTP1/GSTM1 in relation to lung cancer.

${ }^{4}$ Comparison between GSTP1 (AA)/GSTT1 (+) and the other combined genotypes of GSTP1/GSTT1 in relation to lung cancer.

took higher risk of lung cancer as compared to those with genotypes of NAT2 slow, both GSTM1 and GSTT1 present and GSTP1(AA) wild types (OR = $5.500,95 \% \mathrm{CI}=1.219-24.813)$ (Table 5).

\section{Discussion}

GST and NAT2 metabolize many environmental and therapeutic agents affecting susceptibility to several diseases. Polymorphisms of these enzymes result in different host phenotypes and contribute to different disease profiles or response to toxic or therapeutic agents. These correlations are various according to their frequencies in different population. Polymorphisms of GST and NAT2 have been investigated in various populations, including Chinese population. A number of studies tried to explore the correlations among genetic polymorphisms of GSTs or NATs and the risk of lung cancer in different ethnic background and level of exposure factors. The come outs of these studies seemed conflicting [15,37-42]. The reason of these discrepancies is likely that these studies were conducted in diverse populations with different hereditary background and life style or habits (e.g. smoking). As a developing country, China has the peculiarities of explosive population and polluted environment (especially air pollution). Therefore, analysis of the correlation between genetic polymorphisms of phase II metabolic enzymes and lung cancer susceptibility in the population of Central South China was based on our consideration of the same race and the same living environment but not on personal habit of hygiene, such as smoking.

Previous studies demonstrated that GSTM1(-) genotype was significantly correlated with lung cancer in Chinese population, but GSTT1(-) and GSTP1 mutant (AG or GG) genotypes were not $[43,44]$. Also, both NAT2 slow acetylator genotype and homozygous rapid acetylator genotype were reported that are correlated 
Table 3

Combined effects of GSTM1, GSTT1 and GSTP1 genotypes in lung cancer

\begin{tabular}{lcccc}
\hline & M1 (+), T1 (+), P1 (AA) & \multicolumn{2}{c}{$\mathrm{M} 1(-), \mathrm{T} 1(-), \mathrm{P} 1(\mathrm{AG}+\mathrm{GG})$} \\
\cline { 3 - 5 } & $\mathrm{a}$ & $\mathrm{b}$ & $\mathrm{c}$ & $\mathrm{d}$ \\
\hline Cases $(\%)$ & $13(13.4)$ & $32(33.0)$ & $38(39.2)$ & $14(14.4)$ \\
Controls $(\%)$ & $44(22.3)$ & $88(44.7)$ & $55(27.9)$ & $10(5.1)$ \\
OR & & 1.231 & 2.338 & 4.738 \\
$95 \%$ CI & & $0.588-2.578$ & $1.111-4.922$ & $1.708-13.147$ \\
P & & 0.582 & 0.023 & 0.002 \\
$\chi^{2}$ & 0.304 & 5.133 & 9.592 \\
\hline
\end{tabular}

a: GSTM1 (+), GSTT1 (+) and GSTP1 (AA) genotypes.

b: Any one of GSTM1 (-), GSTT1 (-) and GSTP1 (AG+GG) genotypes.

c: Any two of GSTM1 (-), GSTT1 (-) and GSTP1 (AG+GG) genotypes.

d: All of GSTM1 (-), GSTT1 (-) and GSTP1 (AG+GG) genotypes.

Table 4

Designation and frequency of NAT2 alleles in lung cancer

\begin{tabular}{|c|c|c|c|}
\hline & Genotype & Cases $(\%)$ & Controls (\%) \\
\hline NAT2 & & 97 & 197 \\
\hline Rapid acetylators & subtotal & $79(81.4)$ & $154(78.2)$ \\
\hline Homozygous & subtotal & $26(26.8)$ & $46(23.4)$ \\
\hline \multirow[t]{3}{*}{ fast acetylators } & NAT $2 * 4 / * 4$ & 0 & $41(20.8)$ \\
\hline & NAT $2 * 13 / * 13$ & 0 & $2(1.0)$ \\
\hline & NAT $2 * 4 / * 13$ & $26(26.8)$ & $3(1.5)$ \\
\hline Heterozyous & subtotal & $53(54.6)$ & $108(54.8)$ \\
\hline \multirow{12}{*}{ fast acetylators } & NAT $2 * 4 / * 5$ A & 0 & 0 \\
\hline & NAT $2 * 4 / * 5 B$ & $2(2.1)$ & $6(3.0)$ \\
\hline & NAT $2 * 4 / * 5 \mathrm{C}$ & $1(1.0)$ & $2(1.0)$ \\
\hline & NAT $2 * 4 / * 6$ A & $23(23.7)$ & $40(20.3)$ \\
\hline & NAT $2 * 4 / * 6 B$ & 0 & $1(0.5)$ \\
\hline & NAT $2 * 4 / * 7$ A & $2(2.1)$ & $3(1.5)$ \\
\hline & NAT $2 * 4 / * 7 B$ & $22(22.7)$ & $39(19.8)$ \\
\hline & NAT $2 * 4 / * 11 \mathrm{~A}$ & $1(1.0)$ & $1(0.5)$ \\
\hline & NAT $2 * 13 / * 6$ A & 0 & $7(3.6)$ \\
\hline & NAT $2 * 13 / * 7 B$ & $2(2.1)$ & $5(2.5)$ \\
\hline & NAT $2 * 13 / * 5 B$ & 0 & $1(0.5)$ \\
\hline & NAT $2 * 7 \mathrm{~B} / * 12 \mathrm{C}$ & 0 & $3(1.5)$ \\
\hline \multirow[t]{18}{*}{ Slow acetylators } & subtotal & $18(18.6)$ & $43(21.8)$ \\
\hline & NAT $2 * 5 B / * 5 B$ & $1(1.0)$ & 0 \\
\hline & NAT $2 * 5 B / * 7 B$ & $1(1.0)$ & $1(0.5)$ \\
\hline & NAT $2 * 6 \mathrm{~A} / * 5 \mathrm{~B}$ & $3(3.1)$ & $3(1.5)$ \\
\hline & NAT $2 * 6 \mathrm{~A} / * 7 \mathrm{~B}$ & $7(7.2)$ & $12(6.1)$ \\
\hline & NAT $2 * 6 \mathrm{~A} / * 6 \mathrm{~A}$ & $3(3.1)$ & $6(3.0)$ \\
\hline & NAT $2 * 6 \mathrm{~A} / * 6 \mathrm{~B}$ & 0 & $4(2.0)$ \\
\hline & NAT $2 * 6 B / * 6 B$ & 0 & $2(1.0)$ \\
\hline & NAT2 *6A/282.481 & 0 & $2(1.0)$ \\
\hline & NAT2 $* 6 B / 282.481$ & 0 & $1(0.5)$ \\
\hline & NAT $2 * 6 B / * 7 A$ & $1(1.0)$ & $3(1.5)$ \\
\hline & NAT $2 * 6 \mathrm{~B} / * 7 \mathrm{~B}$ & $2(2.1)$ & $2(1.0)$ \\
\hline & NAT2 $* 6 E / 282.481$ & 0 & $1(0.5)$ \\
\hline & NAT2 $* 7 \mathrm{~A} / * 7 \mathrm{~A}$ & 0 & 0 \\
\hline & NAT $2 * 7 \mathrm{~A} / * 7 \mathrm{~B}$ & 0 & $2(1.0)$ \\
\hline & NAT2 *7A/282.481 & 0 & 0 \\
\hline & NAT2 $* 7 \mathrm{~A} / * 11 \mathrm{~A}$ & 0 & $1(0.5)$ \\
\hline & NAT $2 * 7 B / * 7 B$ & 0 & $3(1.5)$ \\
\hline
\end{tabular}

with an increased risk of lung cancer $[16,18]$. These studies mainly focused on establishing the contribution of a single genotype or combination of two genotypes of GST or NAT members to the risk of lung can- cer $[16,45]$, and most of the investigators emphasized to separately investigate the links between genotypes of either GST or NAT family members and lung cancer susceptibility $[3,39,40,46]$. Recently, a few published 
Table 5

Combined effect of NAT2 and GSTs genotypes in lung cancer

\begin{tabular}{lccccc}
\hline & NAT2 (S), M1 (-), T1 (+), P1 (AA) & \multicolumn{3}{c}{ NAT2 (R), M1 (-), T1 (-), P1 (AG+GG) } \\
\cline { 3 - 6 } & $\mathrm{a}$ & $\mathrm{b}$ & $\mathrm{c}$ & $\mathrm{d}$ & $\mathrm{e}$ \\
\hline Cases (\%) & $4(4.1)$ & $15(15.5)$ & $28(28.9)$ & $38(39.2)$ & $12(12.4)$ \\
Controls (\%) & $11(5.6)$ & $49(24.9)$ & $82(41.6)$ & $49(24.9)$ & $6(3.0)$ \\
OR & & 0.842 & 0.939 & 2.133 & 5.500 \\
95\% CI & & $0.234-3.305$ & $0.277-3.187$ & $0.629-7.226$ & $1.219-24.813$ \\
P & & 1.000 & 1.000 & 0.216 & 0.037 \\
$\chi^{2}$ & & 0.000 & 0.000 & 1.529 & 5.241 \\
\hline
\end{tabular}

S: NAT2 slow acetylator, R: NAT2 rapid acetylator.

a: NAT2 slow acetylator, GSTM1 $(+)$, GSTT1 $(+)$ and GSTP1 (AA) genotypes.

b: Any one of NAT2 rapid acetylator, GSTM1 (-), GSTT1 (-) and GSTP1 (AG+GG) genotypes.

c: Any two of NAT2 rapid acetylator, GSTM1 (-), GSTT1 (-) and GSTP1 (AG+GG) genotypes.

d: Any three of NAT2 rapid acetylator, GSTM1 (-), GSTT1 (-) and GSTP1 (AG+GG) genotypes.

e: All of NAT2 rapid acetylator, GSTM1 (-), GSTT1 (-) and GSTP1 (AG+GG) genotypes.

works explored the combination effects of NAT and GST polymorphisms in breast cancer $[47,48]$. These reports documented that some combinations of GST and NAT genotypes possessed an evident dose-gene effect on the occurrence of breast cancer. In present study, we conducted investigation to evaluate the potential correlation between GSTs/NAT2 polymorphisms and the risk of lung cancer, including both separated investigation and combined analysis of GSTM1, GSTT1, GSTP1 and NAT2 genotypes. The results showed that there was no correlation between GSTP1/NAT2 genotypes and the risk of lung cancer, but GSTM1(-) and GSTT1(-) genotypes were correlated with an elevated risk of lung cancer (1.968 fold and 2.046 fold, respectively) when they were analyzed separately. On the other hand, combined analysis of GSTM1, GSTT1 and GSTP1 revealed that the risk of lung cancer of individuals with genotypes of any two of GSTM1(-), GSTT1(-) and GSTP1 mutant (AG+GG) combination was 2.338 fold, whereas with the genotypes of all of GSTM1(-), GSTT1(-) and GSTP1 mutant (AG+GG) combination was 4.738 fold over the controls. Hence, it is obvious that correlation between the risk of lung cancer and the genotypes of either the combination of two GST mutants or the combination of these three GST mutants is more prominent than that of anyone alone. Moreover, a higher lung cancer risk was found when the NAT2 rapid genotype was combined with any two (by 2.133 fold) or three (by 5.500 fold) of GSTM1(-), GSTT1(-) and GSTP1 mutant $(\mathrm{AG}+\mathrm{GG})$ genotypes.

The incidence of lung cancer has been increasing in very recent years. Several factors could be count in that cause this change. First of all is the improvement of the medicare situation and the molecular and pathological techniques. This change enables more patients to be diagnosed. Second is due to the changes of environmental factors, such as smoking, ETS, diet and cooking, pollution, occupational exposure, and other environmental factors. These changes have probably induced a true increase in incidence of lung cancer.

Cigarette smoking is one major environmental factor that causes lung cancer. The role of cigarette smoking in the etiology of the disease is widely accepted and, in the West countries, trends in lung cancer have closely paralleled trends in smoking [49]. However, epidemiological data suggest that there are important ethnic and geographic differences in the relationship between smoking and lung cancer. The observed differences in risk indicate that risk factors, including genetic factors and environmental factors, apart from cigarette smoking, influence individual susceptibility to lung cancer.

Air pollution is one of the environmental causes of lung cancer. Air pollution is a mixture of solid particles, liquids, and gases, each varying in size, composition, and original source [50-52]. Indoor air pollution due to burning, cooking and smoking has been implicated in the genesis of lung cancer. Chinese people traditionally like to make diet by burning wood, smoky coal or gas. Therefore exposure to inhalants, including smoke from combustion and the cooking fumes, is of particular significance in the Chinese population, especially the Han group in Hunan Province because they cook meat and hot cayenne pepper using oil at high temperature everyday. Furthermore, there has been a large population exposed to passive smoking because of many Chinese smokers smoking in living room, bed room and even in the shared offices and buses. It has been indicated that ETS is a mixture of nearly 5000 chemical compounds, including at least 43 known human or animal carcinogens [53]. It is not surprising that ETS causes the same diseases as active smoking, such as lung cancer, although the risk is reduced in proportion due to the dilution in the environment. The observation that nonsmokers may expose to ETS outside 
the home indicates that carcinogens have no threshold and ETS may cause lung cancer in exposed nonsmokers [54]. Outdoor air pollution caused by high level of industrial and constructional pollution has led to the increasing rates of lung cancer in the south area of China recently, where the economic development is in high rates. The airborne particulates contain soot, acid condensates, sulfate, and nitrate particles and can be deeply inhaled. It can be concluded from above that either indoor or outdoor air pollution increases the incidence of lung cancer $[55,56]$.

Lipid may also accelerate the development of lung cancer. The results of a case-control study revealed that there is a correlation between risks of lung cancer and consumption of dietary cholesterol. It has been reported that high animal-fat consumption results in higher risk of lung cancer in females [57]. Also, high fat intake, especially high cholesterol intake has been demonstrated to be associated with an increased risk of lung cancer [58]. The similar results also were reported in other studies [59,60]. Several years ago, Sugita et al. [61] forecasted that the incidence of lung cancer in Chinese population would increase in the future because of economic growth. So far, the Chinese people have been changing their lifestyle to eat more animalfat with the progress of socioeconomic status. It may also be the one of the causes of increasing lung cancer incidence in Chinese people, including Hunan population.

Genetic factor is another import factor that influences the risk of lung cancer. A genetic predisposition to lung cancer is suggested by the observation that only $10-15 \%$ of smokers develop lung cancer. The differences in genotype and allele frequencies suggest the possibility of differences in susceptibility to exposure to environmental carcinogens.

In general, mechanisms by which these agents cause lung cancer are not fully illustrated, but partly due to their effects on the DNA or by promoting the growth of initiated cells.

The toxic and carcinogenic effects of xenobiotic agents are, however, governed by biological uptake and distribution, metabolic activation and detoxification processes and the mechanisms of DNA repair, that are different from individual to individual. Biotransforming toxic xenobiotics to non-toxic form is considered as the first line of defense in the process of the potentially toxic chemical substances towards their subsequent elimination. Therefore, the effective dose received by an individual must be evaluated in terms of the level of biological damage induced by the carcinogenic exposures $[62,63]$.
Carcinogens are metabolized typically in a twophase activation-deactivating sequence. In Phase I, carcinogens were activated mainly by the enzymatic activity from the cytochrome P450 (CYP) family; and in Phase II, usually under the catalyzing of conjugation enzymes, like GST and NAT, the activated carcinogen is processed and rendered more hydrophilic, thus excretable.

Some genetic polymorphisms of metabolic enzymes significantly alter the catalytic activity of the enzyme. GSTM1, GSTT1, GSTP1 and NAT2 are involved in the same biological pathway. The genetic mutations of them produce defective, partially defective enzymes or ones with altered specificities to the substrates. These variations may lead to a reduced Phase II function, which gives rise to higher levels of activated carcinogens. In turn, these activated carcinogens destroy DNA-repair genes, oncogenes and/or tumor suppressor genes by forming DNA adduct. The DNA adduct formation results in accumulated DNA damage, and consequently evokes cell damage and carcinogenesis [24]. Thus, inter-individual variability in xenobiotic metabolism has been associated with different susceptibility to toxicity or cancer risk in response to the same exposure to a given environmental pollutant. Individuals with certain genotypes are inherently more susceptible environmental carcinogens and have higher risk of cancer owing to the increased metabolic activation of carcinogens, decreased detoxification, and/or decreased DNA repair [45,64-66].

In summary, both GSTM1 and GSTT1 polymorphisms may be independent genetic determinants of the risk of lung cancer in non-smoking Chinese population studied in this work. Genetic polymorphisms of GSTP1 and NAT2 exert no independent effect on the risk of lung cancer but they modulate lung cancer risk in combination with GSTM1(-) and GSTT1(-) genotypes. Further studies are required to assess the carcinogenesis role of passive smoking, diet and cooking, pollution, occupational exposure, other environmental factors and potential newer risk factors, in addition to genetic factors, and establish a risk profile for each individual or sub-group in affirmation of the prevention scope.

\section{Acknowledgements}

We thank Dr. LU Yi-qin and Dr. Dong Zi-zheng for their advice and critical reading of our manuscript. 


\section{References}

[1] R. Medeiros, A. Vasconcelos, S. Costa et al., Metabolic susceptibility genes and prostate cancer risk in a southern European population: the role of glutathione S-transferases GSTM1, GSTM3, and GSTT1 genetic polymorphisms, Prostate 58(4) (2004), 414-420.

[2] A. Hirvonen, S.T. Saarikoski, K. Linnainmaa et al., Glutathione S-transferase and $\mathrm{N}$-acetyltransferase genotypes and asbestos-associated pulmonary disorders, J Natl Cancer Inst 88(24) (1996), 1853-1856.

[3] S.J. Lewis, N.M. Cherry, R.M. Niven et al., GSTM1, GSTT1 and GSTP1 polymorphisms and lung cancer risk, Cancer Lett 180(2) (2002), 165-171.

[4] R.C. Strange, B. Matharoo, G.C. Faulder et al., The human glutathione S-transferases: a case-control study of the incidence of the GST1 0 phenotype in patients with adenocarcinoma, Carcinogenesis 12(1) (1991), 25-28.

[5] S. Pemble, K.R. Schroeder, S.R. Spencer et al., Human glutathione S-transferase theta (GSTT1): cDNA cloning and the characterization of a genetic polymorphism, Biochem J 300(1) (1994), 271-276.

[6] J. Brockmoller, I. Cascorbi, R. Kerb et al., Combined analysis of inherited polymorphisms in arylamine $\mathrm{N}$-acetyltransferase 2, glutathione S-transferases M1 and T1, microsomal epoxide hydrolase, and cytochrome P450 enzymes as modulators of bladder cancer risk, Cancer Res 56(17) (1996), 3915-3925.

[7] I. Garcia-Saez, A. Parraga, M.F. Phillips et al., Molecular structure at $1.8 \mathrm{~A}$ of mouse liver class pi glutathione $\mathrm{S}$ transferase complexed with S-(p-nitrobenzyl)glutathione and other inhibitors, J Mol Biol 237(3) (1994), 298-314.

[8] P. Zimniak, B. Nanduri, S. Pikula et al., Naturally occurring human glutathione S-transferase GSTP1-1 isoforms with isoleucine and valine in position 104 differ in enzymic properties, Eur J Biochem 224(3) (1994), 893-899.

[9] M.A. Watson, R.K. Stewart, G.B. Smith et al., Human glutathione S-transferase P1 polymorphisms: relationship to lung tissue enzyme activity and population frequency distribution, Carcinogenesis 19(2) (1998), 275-280.

[10] F. Ali-Osman, O. Akande, G. Antoun et al., Molecular cloning, characterization, and expression in Escherichia coli of fulllength cDNAs of three human glutathione S-transferase Pi gene variants. Evidence for differential catalytic activity of the encoded proteins, J Biol Chem 272(15) (1997), 10004-10012.

[11] D. Ryberg, V. Skaug, A. Hewer et al., Genotypes of glutathione transferase M1 and P1 and their significance for lung DNA adduct levels and cancer risk, Carcinogenesis 18(7) (1997), 1285-1289.

[12] D.W. Hein, M.A. Doll, T.D. Rustan et al., Metabolic activation and deactivation of arylamine carcinogens by recombinant human NAT1 and polymorphic NAT2 acetyltransferases, Carcinogenesis 14(8) (1993), 1633-1638.

[13] D. Hickman, A. Risch, V. Buckle et al., Chromosomal localization of human genes for arylamine $\mathrm{N}$-acetyltransferase, Biochem J 297(3) (1994), 441-445.

[14] D.W. Hein, M.A. Doll, A.J. Fretland et al., Molecular genetics and epidemiology of the NAT1 and NAT2 acetylation polymorphisms, Cancer Epidemiol Biomarkers Prev 9(1) (2000), 29-42.

[15] S.M. Hou, D. Ryberg, S. Falt et al., GSTM1 and NAT2 polymorphisms in operable and non-operable lung cancer patients, Carcinogenesis 21(1) (2000), 49-54.

[16] I. Cascorbi, J. Brockmoller, P.M. Mrozikiewicz et al., Homozygous rapid arylamine $\mathrm{N}$-acetyltransferase (NAT2) geno- type as a susceptibility factor for lung cancer, Cancer Res 56(17) (1996), 3961-3966.

17] T. Oyama, T. Kawamoto, T. Mizoue et al., N-acetylation polymorphism in patients with lung cancer and its association with p53 gene mutation, Anticancer Res 17(1B) (1997), 577-581.

[18] A. Seow, B. Zhao, W.T. Poh et al., NAT2 slow acetylator genotype is associated with increased risk of lung cancer among non-smoking Chinese women in Singapore, Carcinogenesis 20(9) (1999), 1877-1881.

[19] Y. Cao, H. Chen, X. Liu et al., Study on the Relationship between the Genetic Polymorphisms of GSTM1 and GSTT1 Genes and Lung Cancer Susceptibility in the Population of Hunan Province of China, Life Science Research 8(2) (2004), 126-132.

[20] J.A. Moscow, C.R. Fairchild, M.J. Madden et al., Expression of anionic glutathione-S-transferase and P-glycoprotein genes in human tissues and tumors, Cancer Res 49(6) (1989), 14221428.

[21] S. Anttila, L. Luostarinen, A. Hirvonen et al., Pulmonary expression of glutathione S-transferase M3 in lung cancer patients: association with GSTM1 polymorphism, smoking, and asbestos exposure, Cancer Res 55(15) (1995), 3305-3309.

[22] G.W. Mainwaring, S.M. Williams, J.R. Foster et al., The distribution of theta-class glutathione S-transferases in the liver and lung of mouse, rat and human, Biochem J 318(1) (1996), 297-303.

[23] M. Debiec-Rychter, S.J. Land and C.M. King, Histological localization of acetyltransferases in human tissue, Cancer Lett 143(2) (1999), 99-102.

[24] D.P. Miller, G. Liu, I. De Vivo et al., Combinations of the variant genotypes of GSTP1, GSTM1, and p53 are associated with an increased lung cancer risk, Cancer Res 62(10) (2002), 2819-2823.

[25] K. Josen, R. Siegel and D. Kamp, Incidence and Etiology, Shifting Patterns. Cancer of the Lung, From Molecular Biology to Treatment Guidelines, Edited by Alan B. Weitberg. Humana Press, 2002, 3-34.

[26] A. Charloux, E. Quoix, N. Wolkove et al., The increasing incidence of lung adenocarcinoma: reality or artefact? A review of the epidemiology of lung adenocarcinoma, Int $J$ Epidemiol 26(1) (1997), 14-23.

[27] Z.Y. Xu, W.J. Blot, H.P. Xiao et al., Smoking, air pollution, and the high rates of lung cancer in Shenyang, China, $J$ Natl Cancer Inst 81(23) (1989), 1800-1806.

[28] A.H. Wu-Williams, X.D. Dai, W. Blot et al., Lung cancer among women in north-east China, Br J Cancer 62(6) (1990), 982-987.

[29] L. Zhong, M.S. Goldberg, Y.T. Gao et al., A case-control study of lung cancer and environmental tobacco smoke among nonsmoking women living in Shanghai, China, Cancer Causes Control 10(6) (1999), 607-616.

[30] X.R. Yang, S. Wacholder, Z. Xu et al., Caporaso NE. CYP1A1 and GSTM1 polymorphisms in relation to lung cancer risk in Chinese women, Cancer Lett 214(2) (2004), 197-204.

[31] D.P. Ng, K.W. Tan, B. Zhao et al., CYP1A1 polymorphisms and risk of lung cancer in non-smoking Chinese women: influence of environmental tobacco smoke exposure and GSTM1/T1 genetic variation, Cancer Causes Control 16(4) (2005), 399-405.

[32] T. Nakajima, E. Elovaara, S. Anttila et al., Expression and polymorphism of glutathione S-transferase in human lungs: risk factors in smoking-related lung cancer, Carcinogenesis 16(4) (1995), 707-711. 
[33] D.W. Hein, Acetylator genotype and arylamine-induced carcinogenesis, Biochim Biophys Acta 948(1) (1988), 37-66.

[34] H.L. Chiou, M.F. Wu, W.P. Chien et al., NAT2 fast acetylator genotype was associated with an increased risk of lung cancer among never-smoking women in Taiwan, Cancer Lett 223(1) (2005), 93-101.

[35] R.C. Brownson, M.C. Alavanja, N. Caporaso et al., Epidemiology and prevention of lung cancer in nonsmokers, Epidemiol Rev 20(2) (1998), 218-236.

[36] A.C. Deitz, W. Zheng, M.A. Leff et al., N-Acetyltransferase2 genetic polymorphism, well-done meat intake, and breast cancer risk among postmenopausal women, Cancer Epidemiol Biomarkers Prev 9(9) (2000), 905-910.

[37] E. Reszka, W. Wasowicz, K. Rydzynski et al., Glutathione S-transferase M1 and P1 metabolic polymorphism and lung cancer predisposition, Neoplasma 50(5) (2003), 357-362.

[38] M. Kihara, M. Kihara and K. Noda, Lung cancer risk of the GSTM1 null genotype is enhanced in the presence of the GSTP1 mutated genotype in male Japanese smokers, Cancer Lett 137(1) (1999), 53-60.

[39] N. Jourenkova-Mironova, H. Wikman, C. Bouchardy et al., Role of glutathione S-transferase GSTM1, GSTM3, GSTP1 and GSTT1 genotypes in modulating susceptibility to smoking-related lung cancer, Pharmacogenetics 8(6) (1998), 495-502.

[40] H. Wikman, S. Thiel, B. Jager et al., Relevance of Nacetyltransferase 1 and 2 (NAT1, NAT2) genetic polymorphisms in non-small cell lung cancer susceptibility, Pharmacogenetics 11(2) (2001), 157-168.

[41] D. Butkiewicz and M. Chorazy, Individual predisposition to lung neoplasm - the role of genes involved in metabolism of carcinogens, Postepy Hig Med Dosw 53(5) (1999), 655-673.

[42] J.D. Hayes and R.C. Strange, Glutathione S-transferase polymorphisms and their biological consequences, Pharmacology 61(3) (2000), 154-166.

[43] Q. Lan, X. He, D.J. Costa et al., Indoor coal combustion emissions, GSTM1 and GSTT1 genotypes, and lung cancer risk: a case-control study in Xuan Wei, China, Cancer Epidemiol Biomarkers Prev 9(6) (2000), 605-608.

[44] J. Wang, Y. Deng, J. Cheng et al., GST genetic polymorphisms and lung adenocarcinoma susceptibility in a Chinese population, Cancer Lett 201(2) (2003), 185-193.

[45] A. Hatagima, Genetic polymorphisms and metabolism of endocrine disruptors in cancer susceptibility, Cad Saude Publica 18(2) (2002), 357-377.

[46] M. Chan-Yeung, K.C. Tan-Un, M.S. Ip et al., Lung cancer susceptibility and polymorphisms of glutathione-S-transferase genes in Hong Kong, Lung Cancer 45(2) (2004), 155-160.

[47] K.M. Lee, S.K. Park, S.U. Kim et al., N-acetyltransferase (NAT1, NAT2) and glutathione S-transferase (GSTM1, GSTT1) polymorphisms in breast cancer, Cancer Lett 196(2) (2003), 179-186.

[48] O.L. van der Hel, P.H. Peeters, D.W. Hein et al., NAT2 slow acetylation and GSTM1 null genotypes may increase postmenopausal breast cancer risk in long-term smoking women, Pharmacogenetics 13(7) (2003), 399-407.

[49] F. Levi, F. Lucchini, E. Negri et al., Trends in mortality from major cancers in the European Union, including acceding countries, in 2004, Cancer 101(12) (2004), 2843-2850.

[50] X. Xu, J. Gao, D.W. Dockery et al., Air pollution and daily mortality in residential areas of Beijing, China, Arch Environ Health 49(4) (1994), 216-222.

[51] D.W. Dockery, C.A. Pope 3rd, X. Xu et al., An association between air pollution and mortality in six US cities, $N$ Engl $J$ Med 329(24) (1993), 1753-1759.

[52] A.J. Cohen and C.A. Pope, Lung cancer and air pollution, Environ Health Perspect 103(Suppl 8) (1995), 219-224.

[53] R.C. Brownson, M.P. Eriksen, R.M. Davis et al., Environmental tobacco smoke: health effects and policies to reduce exposure, Annu Rev Public Health 18 (1997), 163-185.

[54] M.R. Law and A.K. Hackshaw, Environmental tobacco smoke, Br Med Bull 52(1) (1996), 22-34.

[55] A.J. Cohen, Outdoor air pollution and lung cancer, Environ Health Perspect 108(Suppl 4) (2000), 743-750.

[56] A.J. Cohen, Air pollution and lung cancer: what more do we need to know? Thorax 58(12) (2003), 1010-1012.

[57] A.B. Miller and H.A. Risch, Diet and lung cancer, Chest 96(Suppl 1) (1989), 8S-9S.

[58] R.B. Shekelle, A.H. Rossof and J. Stamler, Dietary cholesterol and incidence of lung cancer: the Western Electric Study, Am J Epidemiol 134(5) (1991), 480-484; discussion on 543-544.

[59] E. De Stefani, H. Deneo-Pellegrini, M. Mendilaharsu et al., Dietary fat and lung cancer: a case-control study in Uruguay, Cancer Causes Control 8(6) (1997), 913-921.

[60] M.C. Alavanja, R.C. Brownson and J. Benichou, Estimating the effect of dietary fat on the risk of lung cancer in nonsmoking women, Lung Cancer 14(Suppl 1) (1996), S63-S74.

[61] M. Sugita, T. Izuno, M. Kanamri et al., Per capita gross national product and summarized odds ratio for epidemiologic studies on the relationship between passive smoking and lung cancer, Tokai J Exp Clin Med 23(5) (1998), 235-240.

[62] C.C. Harris, Interindividual variation among humans in carcinogen metabolism, DNA adduct formation and DNA repair, Carcinogenesis 10(9) (1989), 1563-1566.

[63] V.L. Wilson, A. Weston, D.K. Manchester et al., Alkyl and aryl carcinogen adducts detected in human peripheral lung, Carcinogenesis 10(11) (1989), 2149-2153.

[64] M.R. Spitz and M.L. Bondy, Genetic susceptibility to cancer, Cancer 72(Suppl 3) (1993), 991-995.

[65] H. Bartsch and E. Hietanen, The role of individual susceptibility in cancer burden related to environmental exposure, Environ Health Perspect 104(Suppl 3) (1996), 569-577.

[66] M.J. Khoury, From genes to public health: the applications of genetic technology in disease prevention. Genetics Working Group, Am J Public Health 86(12) (1996), 1717-1722. 


\section{Appendix}

1. PCR primers and the amplified fragment size of GSTs and NAT2 genes

\begin{tabular}{|c|c|c|c|}
\hline $\begin{array}{l}\text { Name of } \\
\text { primer }\end{array}$ & Sequence of primer & $\begin{array}{c}\text { Size of } \\
\text { amplified product }\end{array}$ & $\begin{array}{c}\text { Annealing } \\
\text { temperature }\end{array}$ \\
\hline GSTM1-F1 & 5'-TCAGCGGGATTCTTTGTC-3' & 926 bp & $56^{\circ} \mathrm{C}$ \\
\hline GSTM1-R1 & 5'-CACTTGGAGGATGAATGG-3' & & \\
\hline GSTM1-F2 & 5'-GCAGGAAACAAGGTAAAGG-3' & 994 bp & $60^{\circ} \mathrm{C}$ \\
\hline GSTM1-R2 & 5'-AGGCAGTAGAATCGCTTG-3' & & \\
\hline GSTT1-F1 & 5'-CAAGGGTGCCAAGTGTAG-3' & 845 bp & $56^{\circ} \mathrm{C}$ \\
\hline GSTT1-R1 & 5'-TAGAGTCGCCTTACTTGCC-3' & & \\
\hline GSTT1-F2 & 5'-GAGTAGAGGAAAGGGAATGG-3' & $491 \mathrm{bp}$ & $60^{\circ} \mathrm{C}$ \\
\hline GSTT1-R2 & 5'-TGCACGATAGGTCACCTGAG-3' & & \\
\hline GSTP1-F1 & 5'-CCAATACCATCCTGCGTCAC-3' & $717 \mathrm{bp}$ & $58^{\circ} \mathrm{C}$ \\
\hline GSTP1-R1 & 5'-CGTTACTTGGCTGGTTGATG-3' & & \\
\hline NAT2-F1 & 5'-TTTCCTTACAGGGTTCTG-3’' & $675 \mathrm{bp}$ & $58^{\circ} \mathrm{C}$ \\
\hline NAT2-R1 & 5'-TGTCAAGCAGAAAATGCAAGGC-3' & & \\
\hline NAT2-F2 & 5'-TTCTGTACTGGGCTCTGAC-3' & 892 bp & $62^{\circ} \mathrm{C}$ \\
\hline NAT2-R2 & 5'-AGATAATCACAGGCCATCC-3' & & \\
\hline NAT2-F3 & 5'-CACCTTCTCCTGCAGGTGACCG-3' & $141 \mathrm{bp}$ & $58^{\circ} \mathrm{C}$ \\
\hline NAT2-R1 & 5'-TGTCAAGCAGAAAATGCAAGGC-3' & & \\
\hline NAT2-F4 & 5'-TGAGGAAGAGGTTGAAGAAG-3' & $120 \mathrm{bp}$ & $55^{\circ} \mathrm{C}$ \\
\hline NAT2-R3 & 5'-AAGGGTTTATTTTGTTCCTTATTCTAAAT-3' & & \\
\hline
\end{tabular}

2. Mutation sites and restriction fragments of NAT2 gene

\begin{tabular}{llllll}
\hline Mutation & Site & Restriction site change & $\begin{array}{l}\text { PCR primers and } \\
\text { product size (bp) }\end{array}$ & $\begin{array}{l}\text { Restriction fragments } \\
\text { from wild type (bp) }\end{array}$ & $\begin{array}{l}\text { Restriction fragments } \\
\text { from mutant type (bp) }\end{array}$ \\
\hline $\mathrm{T} \rightarrow \mathrm{C}$ & 111 & Gain of $T a q$ I site & F1/R1 675 & 578,97 & $327,251,97$ \\
$\mathrm{G} \rightarrow \mathrm{A}$ & 191 & Loss of $M s p$ I site & F1/R1 675 & $312,93,270$ & 312,363 \\
$\mathrm{C} \rightarrow \mathrm{T}$ & 282 & Loss of FokI site & F2/R2 892 & $62,429,288,95,18$ & $491,288,95,18$ \\
$\mathrm{~T} \rightarrow \mathrm{C}$ & 341 & Gain of AciI site & F3/R1 141 & 141 & 120,21 \\
$\mathrm{~A} \rightarrow \mathrm{C}$ & 434 & Gain of $M s p$ I site & F2/R2 892 & 892 & 213,679 \\
$\mathrm{C} \rightarrow \mathrm{T}$ & 481 & Loss of KpnI site & F2/R2 892 & 636,256 & 892 \\
$\mathrm{G} \rightarrow \mathrm{A}$ & 590 & Loss of $T a q$ I site & F2/R2 892 & $142,226,170,354$ & $142,396,354$ \\
$\mathrm{C} \rightarrow \mathrm{T}$ & 759 & Loss of $T a q$ I site & F2/R2 892 & $142,226,170,354$ & $142,226,524$ \\
$\mathrm{~A} \rightarrow \mathrm{G}$ & 803 & Gain of DdeI site & F4/R3 120 & 120 & 97,23 \\
$\mathrm{~A} \rightarrow \mathrm{C}$ & 845 & Gain of DraIII site & F2/R2 892 & 892 & 630,262 \\
$\mathrm{G} \rightarrow \mathrm{A}$ & 857 & Loss of BamHI site & F2/R2 892 & 636,256 & 892 \\
\hline
\end{tabular}




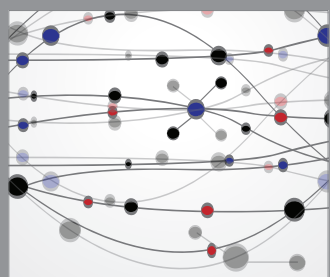

The Scientific World Journal
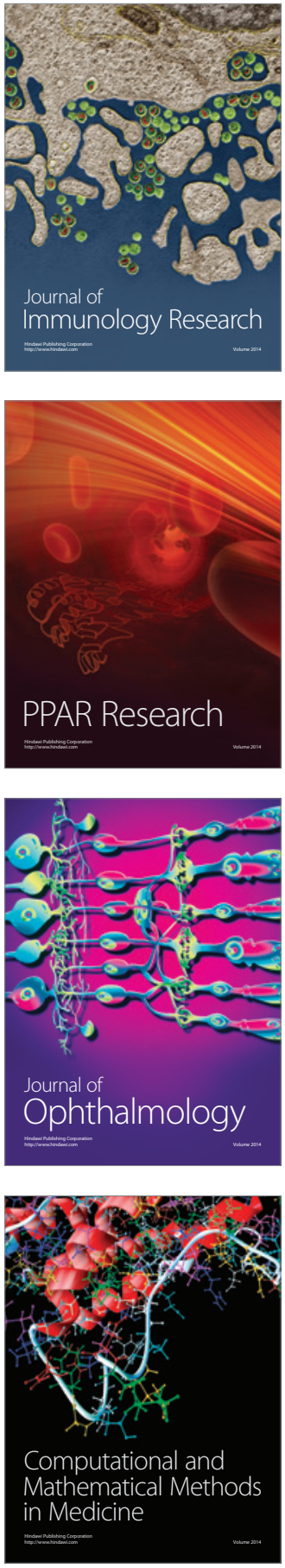

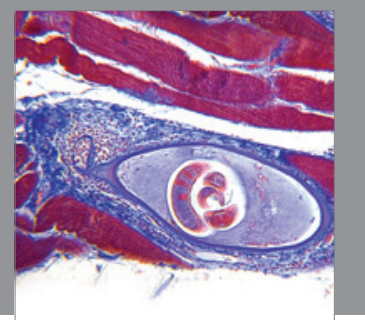

Gastroenterology

Research and Practice
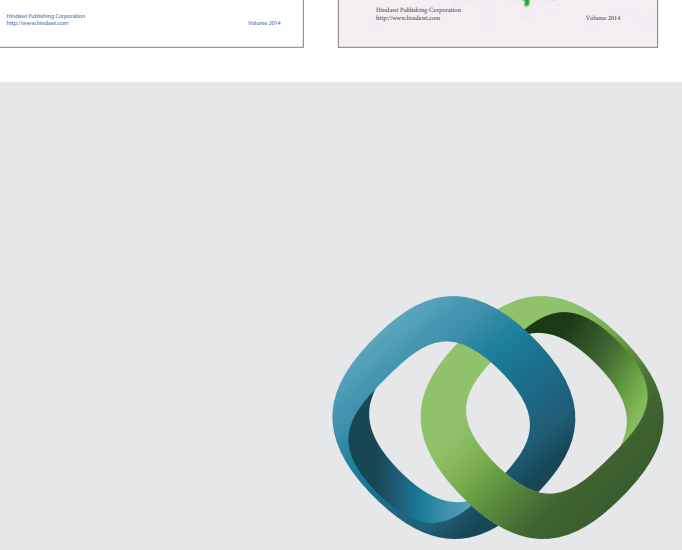

\section{Hindawi}

Submit your manuscripts at

http://www.hindawi.com
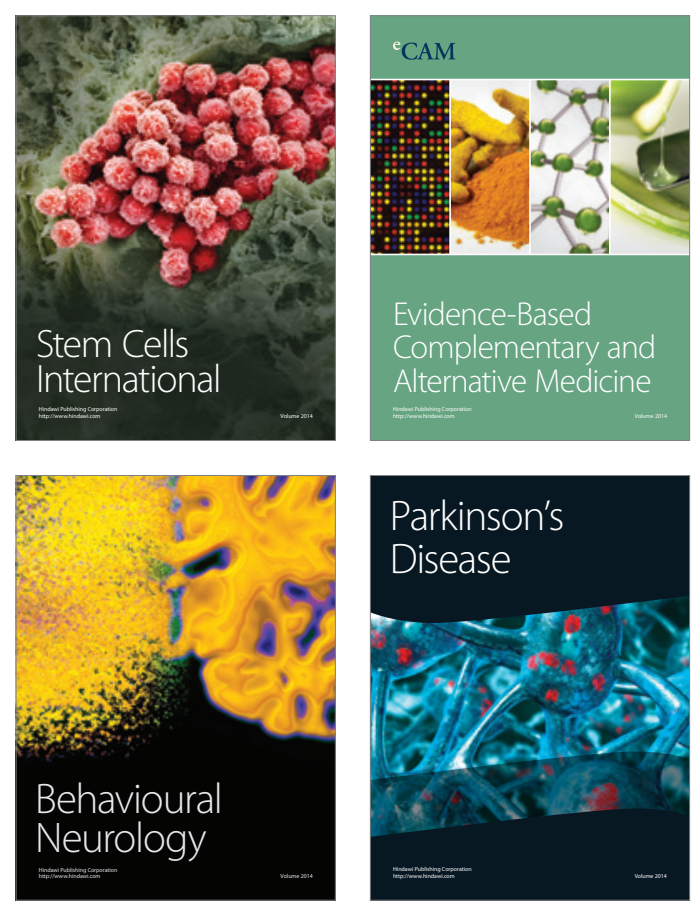

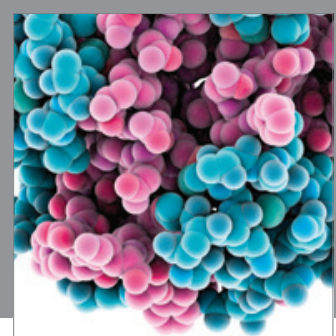

Journal of
Diabetes Research

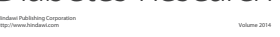

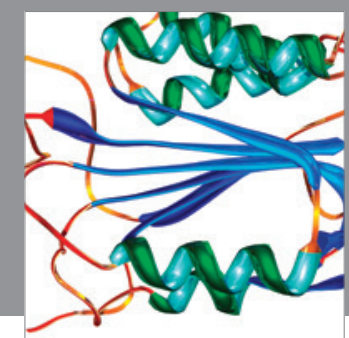

Disease Markers
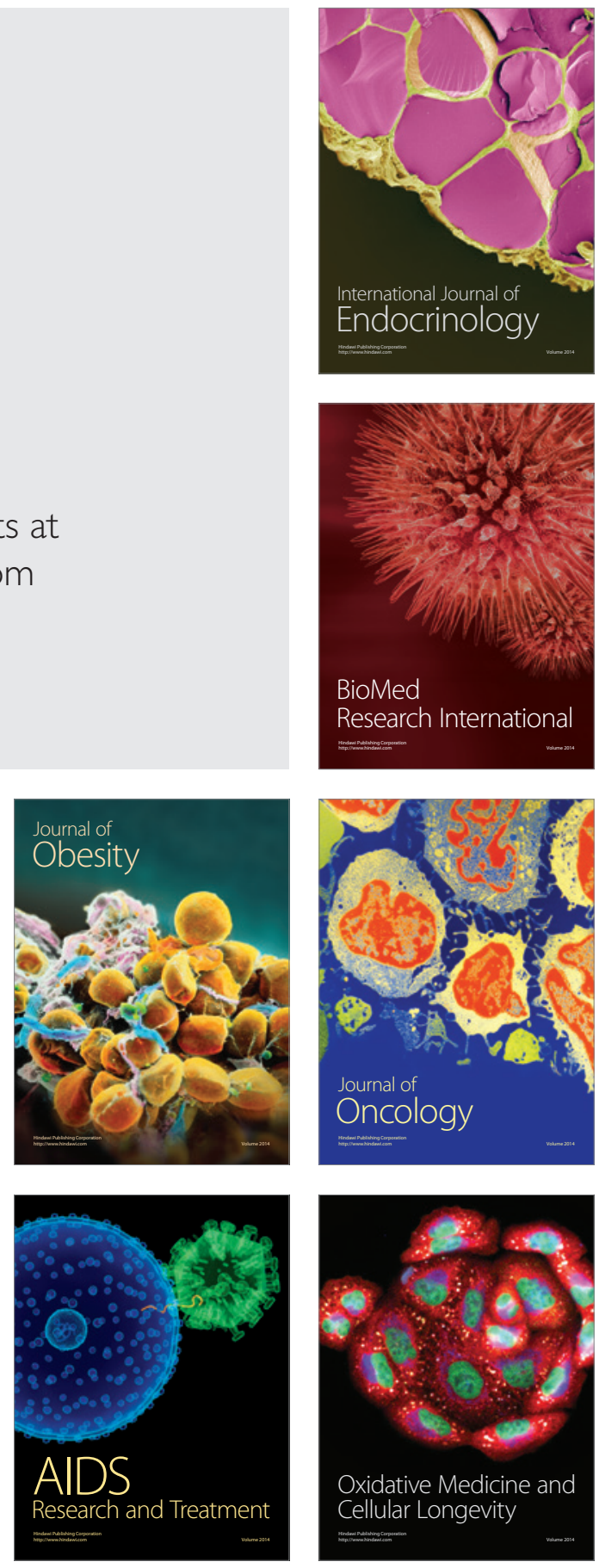